\title{
Fuzzy type-2 applications to analytical chemistry
}

\author{
Zahra Shabaninia*, Hava Torabi
}

Shiraz University, Iran

Email address:

shabani@shirazu.ac.ir (Z. Shabaninia)

\section{To cite this article:}

Zahra Shabaninia, Hava Torabi. Fuzzy Type-2 Applications to Analytical Chemistry. Modern Chemistry. Vol. 1, No. 2, 2013 , pp. $18-21$. doi: $10.11648 /$ j.mc.20130102.11

\begin{abstract}
A general overview about the usefulness of the theory of fuzzy type-2 for solving problems in the field of data analysis and analytical reasoning in analytical chemistry is given. Results of the development of Intel-systems for automated qualitative analysis in X-ray fluorescence analysis and in ICP-atomic emission spectroscopy are discussed.
\end{abstract}

Keywords: Fuzzy Type-2, Analytical Chemistry

\section{Introduction}

There is an increasing interest in Analytical Chemistry in developing intelligent systems that approach human analytical reasoning as close as possible and that enable analytical knowledge to be acquired automatically.

Besides artificial intelligent techniques based on symbolic programming these goals can be pursued by applying the theory of fuzzy sets for matching the analyst's way of reasoning and by neural networks to automate knowledge acquisition and interpretation. There is intensive research going on to combine techniques of fuzzy sets and neural networks, or handle these two methods separately [1].

It can be shown that basic operations of the theory of fuzzy sets can be used in analytical chemistry to solve problems, such as library searching, depth profile comparison or calibration with errors in signals and concentrations. The major area of applications for fuzzy logic, however, concerns the development of expert or intelligent systems, e.g. for spectra interpretation or reasoning in chemical data bases. An example for fuzzy reasoning is given here for rule building in an expert system for automated qualitative analysis with X-ray fluorescence spectroscopy.

Neural networks are studied in analytical chemistry with respect to pattern recognition, modeling and prediction, e.g. in multi component analysis or process control, to classification, clustering or pattern association. Based on the latter technique recent advances are reported for developing an expert system for qualitative ICP-atomic emission spectroscopy. The present paper will use fuzzy type-2 applications to analytical chemistry.

The organization of this paper is as follow. Section II describes the Type-2 Fuzzy Logic overview. Section III de- scribes the applications of type-2 to analytical chemistry and Section IV presets the conclusions.

\section{Type-2 Fuzzy Logic: Overview}

Zadeh introduced fuzzy systems in 1965 and type-2 fuzzy sets in 1975 [2]. So, after 1975, it became necessary to distinguish between pre-existing fuzzy systems and type-2 fuzzy systems; hence, it became common to refer to the preexisting FSs as "T1 FSs" and type-2 fuzzy systems as "T2 FSs" [3]. T1 FSs have been successfully used in many applications $[4,5]$. However, such FSs have limited capabilities to directly handle data uncertainties, where handle means to model and minimize the effect of uncertainties.

Of course, uncertainty comes in many forms and is independent of the kind of FS or methodology one uses to handle it. Two important kinds of uncertainties are linguistic and random. The former is associated with words, and the fact that words can mean different things to different people, and the latter is associated with unpredictability. Probability theory is used to handle random uncertainty and FSs are used to handle linguistic uncertainty, and sometimes FSs can also be used to handle both kinds of uncertainty, because a fuzzy system may use noisy measurements or operate under random disturbances. Within probability theory, one begins with a probability density function (pdf) that embodies total information about random uncertainties. However, in most practical applications, it is impossible to know or determine the pdf; so, the fact that a pdf is completely characterized by all of its moments is used. Unfortunately, it is not possible, in practice, to determine an infinite number of moments; so, instead, at the very least, two moments are used - the mean and variance. Just as variance 
provides a measure of dispersion about the mean, a fuzzy set also needs some measure of dispersion to capture more about linguistic uncertainties than just a single membership function (MF), which is all that is obtained when a T1 FS is used. A T2 FS provides this measure of dispersion.

In type-2 fuzzy logic, the antecedent or consequent membership functions are type-2 fuzzy sets. Such sets are fuzzy sets whose membership grades themselves are type-1 fuzzy sets; they are very useful in circumstances where it is difficult to determine an exact membership function for a fuzzy set; that's why it is said they are useful for incorporating uncertainties [6].

If all uncertainty disappears, then a T2 FS reduces to a T1 FS, as can be seen in Figure 1, e.g. if the uncertainties about the left- and right-end points disappear, then only the dashed triangle survives. This is similar to what happens in probability, when randomness degenerates to determinism, in which case the pdf collapses to a single point. In brief, a T1 FS is embedded in a T2 FS, just as determinism is embedded in randomness [7].

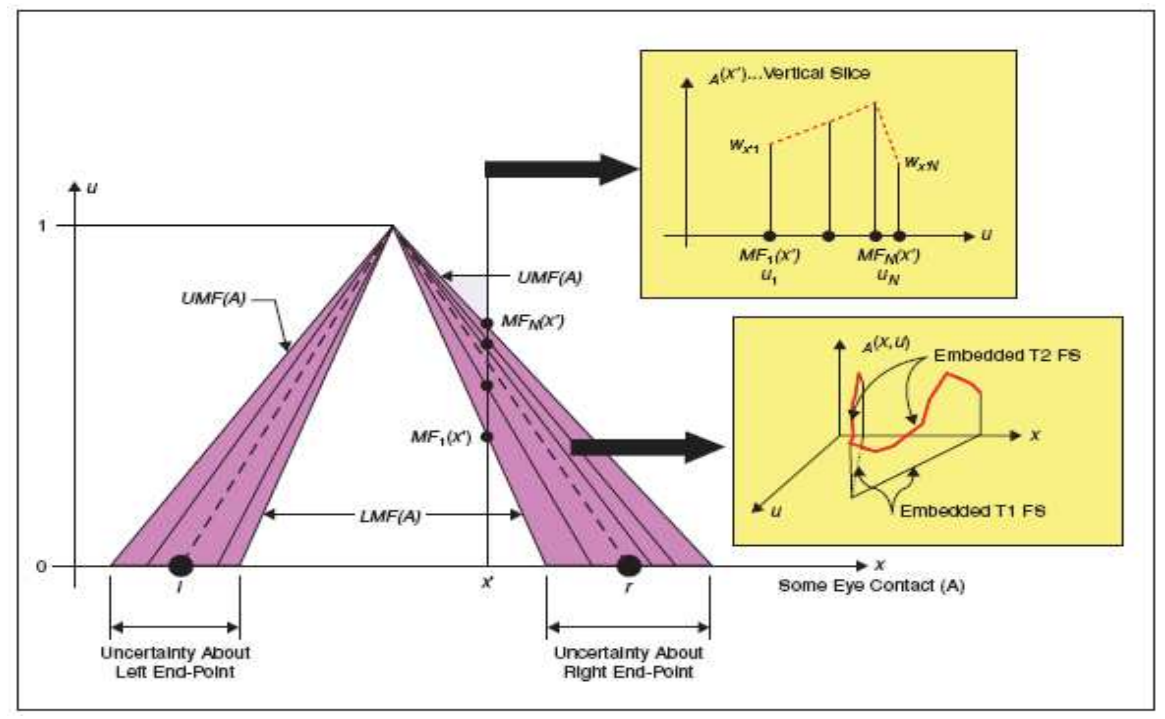

Figure 1. Triangular MFs when base end points have uncertainty intervals.

It is not as easy to sketch 3-D figures of a type-2 membership function (T2 MF). Another way to visualize a T2 FS is to sketch (plot) its footprint of uncertainty (FOU) on the 2-D domain of the T2 FS, and this is easy to do. The heights of a T2 MF (its secondary grades) sit atop its FOU.

In Figure 1. If the continuum of triangular MFs is filled in (as implied by the shading), then the FOU is obtained. Another example of an FOU is shown in Figure 2. It is for a Gaussian primary MF whose standard deviation is known with perfect certainty, but whose mean, $\mathrm{m}$, is uncertain and varies anywhere in the interval from $\mathrm{m} 1$ to $\mathrm{m} 2$. The uniform shading over the entire FOU means that uniform weighting (possibilities) is assumed. Because of the uniform weighting, this T2 FS is called an interval type-2 FS (IT2 FS) [7].

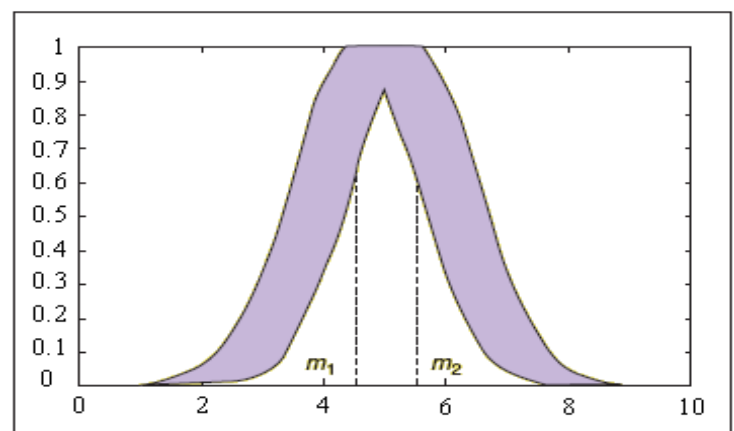

Figure 2. FOU for a Gaussian primary MF whose mean varies in the interval $\left[m_{1}, m 2\right]$ but having constant standard deviation..

\section{Applications}

With this overview analytical problems have been solved with respect to multi criteria decision making [8] or pattern matching in spectroscopy or chromatography [9].

T. George applied the fundamental fuzzy set operations in an interpretation system for automated qualitative analysis in X-ray fluorescence spectroscopy [10]. The measured Xray fluorescence spectrum is transferred to a line spectrum by using the $2^{\text {nd }}$ derivative computed by a Savitzky-Golay digital filter and the resulting lines are fuzzified using type2 according to their variability (Figure3).

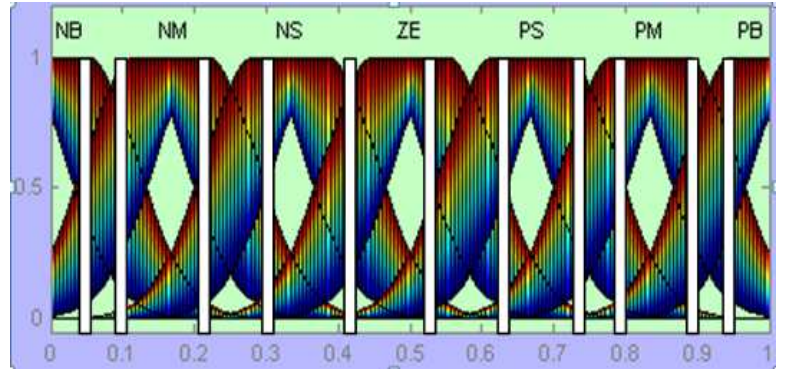

Figure 3. Comparison of a fuzzified sample spectrum with a crisp candidate reference spectrum by fuzzy type 2 intersection.

In the next step the membership functions have to be unified as demonstrated in Fig. 3 by bold lines covering the membership functions. Comparison of the measured spec- 
trum to the library spectra of the elements from sodium (atomic number 11) to uranium (atomic number 92) is performed by fuzzy intersection. In this approach the library spectrum is assumed to be crisp. As the result of intersection discrete membership values are obtained that are aggregated by calculating the relative cardinality, i.e. the sum of the membership values normalized to the number of lines in the candidate reference spectrum. To aid analytical reasoning the theory of fuzzy sets is used in the sense of fuzzy logic and approximate reasoning. Fuzzy logical operations are defined for all the logical connectives known from classical (Boolean) logic, such as AND, OR, NOT or the implication. In most applications a fuzzy logical AND is defined by intersection, a fuzzy OR by the union, the NOT by the fuzzy complement and an implication (IF $\mathrm{V}=\mathrm{A}$ THEN $\mathrm{U}=\mathrm{B}$ ) by the membership function over the Cartesian product A x B represented by the minimum between the m.f.'s of A and B. Fuzzy logic has been mainly applied until now in the context of fuzzy control. In chemistry this idea has been used by Yamada [11] to control fermentation of coenzyme $\mathrm{Q}_{10}$ based on monitoring cell mass with a turbid metric sensor. In this way the very complex process that cannot be described by a mathematical model is controlled by some rules of thumb specified by fuzzy sets. In the above mentioned example of X-ray fluorescence analysis fuzzy logic can be used to specify the following types of rules:

- The intensity ratio of $\mathrm{Ka}$ and $\mathrm{K}_{\mathrm{b}}$-lines should be "about 5".

- For elements with atomic numbers less than 20 the detection probability of $\mathrm{K}_{\mathrm{b}}$-lines is "decreased heavily".

- Resolution of $\mathrm{L}_{\mathrm{b} 1}$ and $\mathrm{L}_{\mathrm{b} 5}$ lines "decreases with increasing atomic Numbers".

These rules are formalized in the usual manner as implications, e.g. IF the line pair is $\mathrm{K} \mathrm{a}, \mathrm{K}_{\mathrm{b}}$ THEN intensity ratio is "about 5". The data "about 5" is represented here as a fuzzy number and the degree of membership of a measured intensity ratio is inferred by applying Zadeh's compositional rule of inference [12]. Different schemes of reasoning can be applied, for example the theory of approximate reasoning as further developed by Yager [13]. Approximate reasoning has been already explored in analytical chemistry for reasoning about missing data/information in a data base on $\mathrm{pH}$ indicators [14] and is used at present for building an interpretation system in IR- spectroscopy. The concept of fuzzy theory is also used for data analysis in analytical chemistry. A difference to statistical data analysis can be understood as follows: a statistical (probabilistic) approach describes the set of potential outcomes on the basis of a certain distribution. The observation results are vague only before experimentation but are a determined object after sampling. In a fuzzy-set theoretic (possibility) approach the concrete observation is available only with certain vagueness either due to the uncertainty of the data themselves or due to our description of the observed object by linguistic expressions. Because both types of observations can be specified as fuzzy sets data analysis can be carried out independent on the observation type, Up to now fuzzy data analysis has been applied to univariate and multivariate modeling [9], to pattern recognition problems [15-17] or to clustering of data [18]. For example, grouping of malt samples analyzed for 9 physic-chemical parameters was performed on the basis of fuzzy clustering [18]. The advantage of fuzzy methods of clustering over conventional crisp cluster algorithms is the feasibility to assign a single object to more than one cluster simultaneously. By means of pattern recognition gasoline samples analyzed by capillary gas chromatography can be matched to typical reference chromatograms of fuels in a library [19].

\section{Conclusion}

In this study, a promising approach using fuzzy type-2 is proposed in analytical chemistry. The proposed architecture utilizes the capabilities of a type- 2 fuzzy architecture in properly handling of uncertainties in computing the output.

\section{References}

[1] Proceedings of the International Conference on Fuzzy Logic \& Neural Networks, Iizuka (Japan), July 22-24 (1 99 9 0). [2] L.A. Zadeh, "The concept of a linguistic variable and its application to approximate reasoning-1", Information Sciences, vol. 8, pp. 199-249, 1975.

[2] J.M. Mendel, "Uncertain Rule-Based Fuzzy Logic Systems: Introduction and New Directions", Prentice-Hall, UpperSaddle River, NJ, 2001.

[3] F. Shabaninia, "Type-2 Fuzzy Multiagent Traffic Signal Control", IEEE-IRI Conference, Las Vegas 2012.

[4] F. Shabaninia, "Fuzzy Type-2 Electrode Position Controls for an Electric Arc Furnace ", IEEE-IRI Conference, Las Vegas 2012.

[5] J. M. Mendel, "Type-2 Fuzzy Sets and Systems: An Overview”, IEEE Computational Intelligence Magazine, 2007.

[6] N. N. Karnik, J. M. Mendel, Q. Liang, "Type-2 Fuzzy Logic Systems", IEEE Transactions on Fuzzy Systems, vol. 7, NO. 6, 1999 .

[7] M. Otto, Chemo metrics Intelligent Lab. SYS. 4, 101-120 (1 $988)$.

[8] H. Bandemer and M. Otto, Mikrochim. Acta [Wien] 11, 93124 (19 98 ).

[9] T. George, M. Otto and W. Wegscheider. Y. Yamada, S. Murayama and S. Shiomi, J. Chem. Eng. Of Japan 24, 94-99 (1 $991)$.

[10] L.A. Zadeh, in J.E. Hayes, D. Michie and L.I. Kulich (Eds.), Machine Intelligence 9, Wiley, New York, 149-194 (1 97 9).

[11] R.R. Yager, Artificial Intel. 31, 99-112 (1 98 7).

[12] M. Otto and R.R. Yager, in G. Gauglitz (Ed.), SoftwareEntwicklungen in der Chemie 3, Springer-Verlag, Berlin Heidelberg. 229-245 11989). 
[13] T.-Blaffert, Anal, 'Chim. Acta 161, 135-148 (1 98 4).

[14] M. Otto and H. Bandemer, Anal. Chim. Acta 184, 21-31 (1 9 $86)$

[15] E. Stottmeister, H. Hermann, P. Hendel, D. Feiler, M. Nagel and H.-J. Dobberkau, Fresenius Zeitschrift f. Anal. Chemie
327, 709-714 (1 98 7).

[16] T. Jacobson, K. Kolset and N.B. Vogt, Mikrochim. Acta [Wienl TI, 125-138 (1 $\left.9 \begin{array}{lll} & 8 & 6\end{array}\right)$.

[17] E.W. Robb and M.E. Munk, Mikrochim, Acta fWienl I, 131155 (1990). 